\title{
Runaway electron mitigation by 3D fields in the ASDEX-Upgrade experiment
}

\author{
M.Gobbin ${ }^{1}$, L.Lii ${ }^{2}$, Y.Q.Liu ${ }^{3}$, L.Marrelli ${ }^{1}$, M.Nocente ${ }^{4}$, G.Papp ${ }^{5}$, G.Pautasso ${ }^{5}$, \\ P.Piovesan ${ }^{1}$, M.Valisa ${ }^{1}$, D.Carnevale ${ }^{6}$, B.Esposito ${ }^{7}$ L.Giacomelli $^{8}$, \\ M.Gospodarczyk ${ }^{6}$, P.J.McCarthy ${ }^{9}$, P.Martin ${ }^{1}$, W.Suttrop ${ }^{5}$, M.Tardocchi ${ }^{8}$, \\ M.Teschke ${ }^{5}$, the ASDEX Upgrade Team ${ }^{5}$ and the EUROfusion MST1 Team* \\ ${ }^{1}$ Consorzio RFX, Padova (CNR, ENEA, INFN, \\ Universitá di Padova, Acciaierie Venete SpA)- Italy \\ ${ }^{2}$ College of Science, Donghua University, Shanghai, Peoples Republic of China \\ ${ }^{3}$ General Atomics, San Diego, CA 92186-5608, USA \\ ${ }^{4}$ Dipartimento di Fisica, Universitá di Milano-Bicocca, Milano, Italy \\ ${ }^{5}$ Max-Planck-Insitute for Plasma Physics, Garching, Germany \\ ${ }^{6}$ Dip. di Ing. Civile ed Informatica, Universitá di Roma Tor Vergata, Italy \\ ${ }^{7}$ ENEA, Fusion and Nuclear Safety Department, Via E. Fermi 45, 00044 Frascati, Italy \\ ${ }^{8}$ CNR-IFP, Milan, Italy \\ ${ }^{9}$ Department of Physics, University College Cork, Cork, Ireland and \\ *see H. Meyer et al., Nuclear Fusion FEC 2016 Special Issue (2017)
}

Disruption-generated runaway electron (RE) beams represent a severe threat for tokamak plasma-facing components in high current devices like ITER, thus motivating the search of mitigation techniques. The application of 3D fields might aid this purpose and recently was investigated also in the ASDEX Upgrade experiment by using the internal active saddle coils (termed B-coils). Resonant magnetic perturbations (RMPs) with dominant toroidal mode number $n=1$ have been applied by the B-coils, in a RE specific scenario, before and during disruptions, which are deliberately created via massive gas injection. The application of RMPs affects the electron temperature profile and seemingly changes the dynamics of the disruption; this results in a significantly reduced current and lifetime of the generated RE beam. A similar effect is observed also in the hard-X-ray (HXR) spectrum, associated to runaway electron emission, characterized by a partial decrease of the energy content below $1 \mathrm{MeV}$ when RMPs are applied. The strength of the observed effects strongly depends on the upper-to-lower B-coil phasing, i.e. on the poloidal spectrum of the applied RMPs, which has been reconstructed including the plasma response by the code MARS-F. A crude vacuum approximation fails in the interpretation of the experimental findings: despite the relatively low $\beta(<0.5 \%)$ of these discharges, a modest amplification (factor of 2 ) of the edge kink response occurs, which has to be considered to explain the observed suppression effects.

PACS numbers: 


\section{INTRODUCTION}

The mitigation of runaway electrons still represents an important challenge in the development of a tokamak fusion reactor and in particular for future operations in ITER [1]. These high energy particles are often generated during disruptions [2-4], large scale macroscopic events in tokamak plasmas that lead to a rapid termination of the discharge. During disruptions the machine is subjected to massive thermal and electromagnetic loads as the thermal energy and current dissipate in a time on the order of few milliseconds. The temporal sequence for these highly deleterious phenomena are often characterized by a precursor phase during which the plasma pressure and current build up to conditions that trigger MHD instabilities followed by the loss of the thermal energy to the first wall (thermal quench). Finally, the ohmic current rapidly falls to zero inducing huge electromechanical forces on plasma facing components (current quench phase). Due to the fast growth of the toroidal electric field during the current quench phase, a fraction of electrons may be continuously accelerated up to velocities close to the speed of light i.e. they undergo a runaway process. Indeed, runaway electrons are generated when the force exerted on the free electrons by the toroidal electric field exceeds the frictional drag due to collisions with the background distribution of electrons (primary RE production) [5]. Moreover, if a runaway electron is subject to a Coulomb collision with a thermal one, the latter may gain enough forward momentum to become, in turn, a runaway. Thereafter, by the same process, both electrons can create even more runaways (secondary RE or avalanche generation [6]).

Thus, RE generation depends both on the applied electric field and on collisionality i.e. the plasma density; if no other RE energy loss mechanisms other than collisional damping are involved, a simple relation holds between the critical value of the electric field (in $\mathrm{V} \mathrm{m}^{-1}$ units) necessary to produce any runaway and the electron density $n_{20}$ in units of $10^{20}\left(\mathrm{~m}^{-3}\right)[7]$ :

$$
E_{\text {crit }}=\frac{n_{e} e^{3} \ln (\Lambda)}{4 \pi \epsilon_{0} m_{e} c^{2}} \approx 0.08 n_{20}
$$

In standard discharges the loop voltage is small and thus the corresponding electric field is below $E_{\text {crit }}$; nevertheless the large electric field generated during disruption events can accelerate the electrons up to energies greater than $10 \mathrm{MeV}$ and the plasma resistive current is replaced by a current of relativistic electrons. In ITER the secondary RE generation will be dominant and significantly different from those experimentally observed up to now; thus an adequate protection plan for ITER is difficult to extrapolate and to be validated in existing tokamaks $[8,9]$. Theoretical calculations predict that the avalanche process could multiply the number of relativistic electrons 
by about twelve orders of magnitude more in ITER than in JET [10]. Most of the post-disruption current is expected to be carried by runaway electrons with an energy up to $\sim 100 \mathrm{MeV}[11]$. These energetic particles can strike and seriously damage the surrounding structures through their highly localized energy deposition. The prevention and mitigation of their harmful effects is thus of paramount importance to ensure a long life to a commercial reactor. For safe operations in ITER the post-disruption runaway current must be kept much below 1MA and the current quench phase duration between $50 \mathrm{~ms}$ and $150 \mathrm{~ms}$ [12].

The main strategies envisaged to mitigate post-disruption generated runaway electrons are based on:

1. methods to increase the plasma density, in order to enhance the critical electric field;

2. deconfinement techniques which reduce the RE loss time;

3. magnetic confinement of the RE current while energy is removed by loop voltage control.

While the methods 1) and 2) act in order to suppress the primary RE generation and avalanche mechanism, the third is based on the runaway beam position control [13, 14]. At present the first one, i.e. the dissipation of the energy of fast electrons via Coulomb scattering by increasing the density, is considered to be the main solution. Several techniques directed to this end are extensively investigated in the tokamak community, for instance Massive Gas Injection (MGI) [15-20] and shattered pellet injection [21, 22].

RE suppression by deconfinement takes advantage of magnetic perturbations to keep magnetic surfaces from healing. This can be obtained both by producing secondary disruptions by dense gas jets injected repeatedly during the CQ phase [23] or by applying external non axi-symmetric fields generated by external coils before the onset of the disruption. In particular this latter approach $[24,25]$ has been tested in existing devices and examined with numerical simulations dedicated to ITER scenarios $[11,26,27]$. While few of these experiments reported promising outcomes [28, 29, 31, 32], attempts in JET showed no effects [20]. In DIII-D a $n=3$ resonant magnetic perturbation [33] was able to partially reduce the post-disruption runaway current, while $n=1$ patterns failed [22]. A major and innovative contribution to these studies comes from the experiments recently performed [34] in the medium size tokamak ASDEX Upgrade [35], here discussed in detail. RMPs applied by external coils [36] before disruption events significantly reduce the current and lifetime of the resulting RE beams with a strength depending on the perturbation poloidal spectrum. It is found, in particular, that the poloidal spectra which give the maximum RE 
current reduction cannot be interpreted in terms of the vacuum field approximation but the plasma response to the RMPs [37] - i.e. the capability of a plasma to amplify magnetic perturbations and hence to experience significant helical deformations - is to be taken into account. Finally, the decrease in the post-disruption RE current is a nonlinear function of the amplitude of selected harmonics of the poloidal spectrum.

In the next section of the paper the baseline runaway scenario in ASDEX Upgrade and the phenomenology of standard discharges, when no perturbations are applied, is presented. Section III reports the effect of RMPs with different poloidal spectrum on the final runaway current. The impact of RMPs on the disruption dynamic, on the thermal quantities and HXR radiation are described in Sections IV-VI. The interpretation of the experimental results by considering the role played by the plasma response to RMPs is illustrated in Section VII. Finally, the effect of magnetic perturbations of reduced amplitude or applied only during the post-disruption phase is investigated in Section VIII. The conclusions are drawn in the last section.

\section{RUNAWAY SCENARIO IN ASDEX UPGRADE}

AUG disruptions, typically of diverted and elongated plasmas, do not exhibit formation of RE beams. Indeed, usually a disrupting plasma has a density large enough to suppress the RE primary generation and avalanche mechanisms are not expected to be significant in AUG; moreover, during the thermal quench, an elongated plasma becomes vertically unstable, moves up or downwards, becomes limited on the lower or upper divertor and develops a region of open flux surfaces (halo) from which the REs escape within milliseconds. Therefore, in order to allow studies of RE generation and mitigation during disruption a dedicated scenario has been established [19].

The ASDEX Upgrade scenario for these experiments is based on a discharge with toroidal magnetic field $B_{T}=-2.5 \mathrm{~T}$, plasma current of $I_{p}=800 \mathrm{kA}$ and central electron density in the range: $2.5-3.5 \cdot 10^{19} \mathrm{~m}^{-3}[19,38]$. The plasma is circularly shaped (Fig.1-(e)), limited by the inner wall; a power of 2.5 MW of Electron Cyclotron Resonance Heating (ECRH) is applied for 100 ms from $t=0.9 \mathrm{~s}$ to heat the plasma and introduce a fast particle seed just before the disruption, which is triggered by the injection of argon gas $\left(1.7-5 \cdot 10^{21}\right.$ atoms $)$ at $t=1 \mathrm{~s}$. The injected argon induces a fast quench of the current driven by thermal electrons, followed by a long-lived runaway beam with a toroidal current up to $400 \mathrm{kA}$ and lasting up to $500 \mathrm{~ms}$. The RE beams are reproducible but changes in the current ramp up history, in the toroidal magnetic field, in the deposited ECRH power, in the reference current imposed by the control system might prevent the 

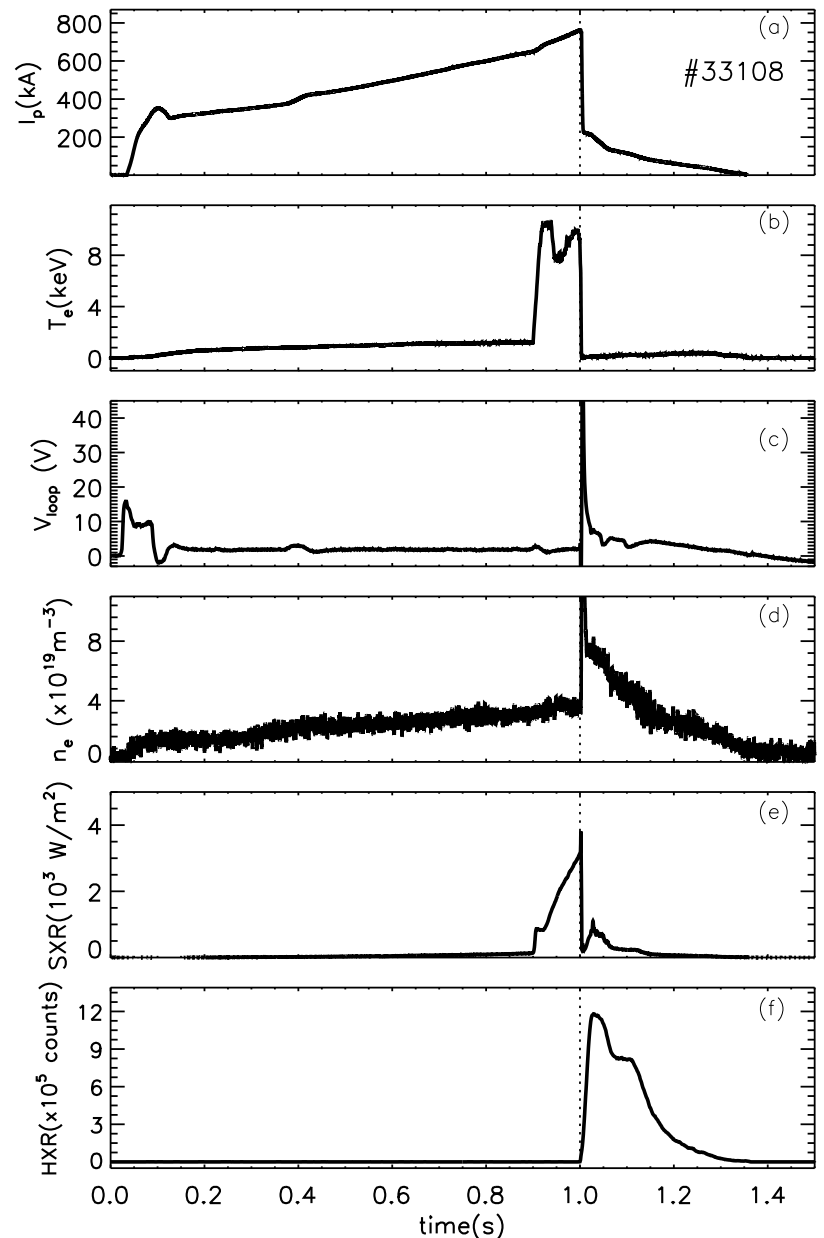

FIG. 1: Evolution of (a) plasma current, (b) core electron temperature (ECE at $R=1.67 \mathrm{~m}, Z=0.024 \mathrm{~m}$ ), (c) loop voltage (MAU-ULid12 signal), (d) electron density at the edge (along H-5 chord), (e) SXR brightness (along line of sight F017 of tomography camera F) (d) HXR emission for the shot \#33108 $\left(\mathrm{LaBr}_{3}(\mathrm{Ce})\right.$ scintillator diagnostic). The dashed vertical black line at 1s corresponds to the trigger time of disruption.

formation of a beam or influence its current and duration. The variation of these conditions must be further exploited and extended.

Fig.1 shows the main waveforms for a typical discharge evolution; the plasma current $I_{p}$ is ramped till $t=1 \mathrm{~s}$, when the disruption is induced. Then, part of $I_{p}$ is converted into runaway beam current $\left(I_{R E}\right)$ with an initial value of $\sim 200 \mathrm{kA}$ decreasing to zero in about $0.35 \mathrm{~s}$. The ramp down of the current is operated by the control system following a pre-programmed time 

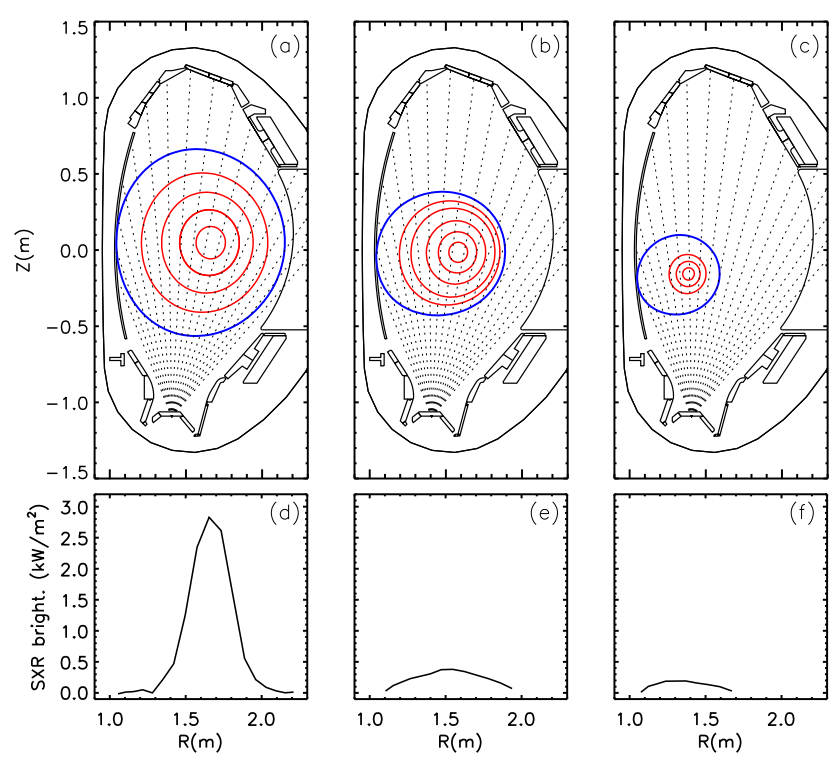

FIG. 2: Reconstruction of plasma equilibria at three different times for the shot \#33108: panel (a) for $\mathrm{t}=0.98 \mathrm{~s}$, panel (b) for $\mathrm{t}=1.1 \mathrm{~s}$, panel (c) for $\mathrm{t}=1.18 \mathrm{~s}$. The dotted lines are the chords of the soft-X-ray tomography camera F. In (d),(e),(f) the associated soft-X-ray radiation as observed from the soft-x-ray tomography camera F.

trace. When larger amounts of argon are injected, the current decay is accelerated and the Ohmic system cannot keep the actual current at the level of the reference value. The central electron temperature measured by the Electron Cyclotron Emission diagnostic grows from 1 to $\sim 10 \mathrm{keV}$ during the ECRH heating and collapses at $1 \mathrm{~s}$ in less than $1 \mathrm{~ms}$ (thermal quench phase, in panel (b)). Before the disruption the loop voltage is below $3 \mathrm{~V}$ but fast increases during the current quench up to $\sim 20 \mathrm{~V}$; then, it decreases again to values around $5 \mathrm{~V}$ in the RE beam phase and finally to zero. Panel (d) shows the electron density $n_{e}$ along an interferometer chord at the edge of the plasma; when argon is injected the density sharply grows by about a factor of $\sim 3$; then during the RE beam phase it is reduced from $8 \cdot 10^{19} \mathrm{~m}^{-3}$, just after the disruption, to zero. In general the argon puff might increase the density of a factor between 2 and 6 . The soft-X-ray radiation from the core of the plasma is reported in panel (e); it basically follows the electron temperature evolution: a fast growth during the ECRH heating and a sudden collapse at the thermal quench phase. Finally, Hard X-Ray (HXR) measurements from a scintillator diagnostic $[39,40]$ are reported in the last panel; they are greater than zero only in the post-disruption phase with a temporal evolution similar to the plasma current in panel (a). 
The evolution of the plasma equilibrium before and after the disruption has been reconstructed by means of the code CLISTE [41] and is shown in Fig.2 for three times: $t=0.98 \mathrm{~s}(\mathrm{a}), t=1.1 \mathrm{~s}$ (b), $t=1.18 \mathrm{mss}$ (c); panels (d)-(e)-(f) display the corresponding SXR profiles along the lines of sight plotted with dotted lines in (a)-(b)-(c). It is worth to note that the code CLISTE is not fully appropriate to describe the post-disruption phases and in particular the RE beam equilibria; a further development of such a code to deal with extreme RE relevant cases is still in progress. Despite these limitations, a reasonable agreement is found between the reconstructed equilibria and the SXR profiles. Before the disruption the average minor radius of the plasma is $a \sim 0.53 \mathrm{~m}$ with a major radius of $R_{0}=1.63 \mathrm{~m}$. Then, $100 \mathrm{~ms}$ after the disruption, the plasma has moved inward and its minor radius is reduced to $a \sim 0.4 \mathrm{~m}$; the associated SXR radiation is decreased by a factor 5. Finally, during the later stage of its evolution, at $t=1.18 \mathrm{~s}$ the RE beam plasma minor radius is further lowered to $a \sim 0.2 \mathrm{~m}$ with SXR radiation reduced by a factor 10 with respect to $t=0.98 \mathrm{~s}$.

\section{APPLICATION OF MAGNETIC PERTURBATIONS BY B-COILS}

ASDEX Upgrade is equipped with a set of sixteen non-axisymmetric in-vessel coils in the form of two toroidal rows of eight coils (termed B-coils) above and below the tokamak midplane on the outer side of the torus (low field side). During the experiments described in this paper, they were powered by independent power supplies which produce a radial field of $\sim 10^{-3} \mathrm{~T}$ at the plasma boundary in front of an upper coil $\left(b^{r} / B_{T} \lesssim 10^{-3}\right.$ for typical $2.5 \mathrm{~T}$ operations). The B-coils can generate resonant magnetic perturbations with dominant toroidal mode numbers $n=1,2,4$. The poloidal mode number spectrum $m$ is defined by the poloidal dimension of the coils and their reciprocal distance; generally there is no single corresponding $m$, but a broad spectrum of modes and harmonics. The differential phase $\Delta \phi$ between the current harmonic flowing in the upper $\left(I_{\text {upper }}\right)$ and lower $\left(I_{\text {lower }}\right)$ set of coils can be modified in order to change the alignment of the perturbation field with respect to the equilibrium magnetic field lines and hence the degree of MP resonance with rational $q$ surfaces. The differential phase $\Delta \phi$ is defined through the following relations: $I_{\text {upper }} \propto \cos \left(n \phi_{\text {coil }}\right)$ and $I_{\text {lower }} \propto \cos \left(n \phi_{\text {coil }}+\Delta \phi\right)$ where $\phi_{\text {coil }}$ is the toroidal angle location of the center of a B-coil. One-side effect of the plasma response to the field perturbations may be a modification of these resonance conditions with respect to a vacuum approximation.

In the experiments reported here a scan of $\Delta \phi$ in steps of $45^{\circ}$ is performed and the perturbations are characterized by a dominant $n=1$ toroidal mode number; a toroidal field of $2.5 T$ limits the 


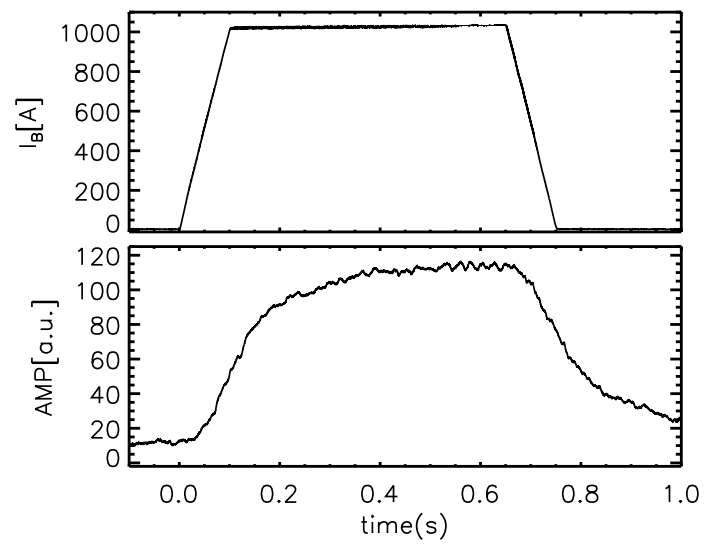

FIG. 3: Upper panel: waveform for the current flowing in one of the B-coil (MAW-IBu1 signal); lower panel: corresponding field in the plasma estimated from high field side probe coils (from shot \#33706, the time scale is shifted of $-0.5 \mathrm{~s}$ with respect to the experimental one, so $t=0 \mathrm{~s}$ coincides with the time at which the B-coil are turned on).

maximum current in the B-coils to $I_{B}=1 \mathrm{kA}$. The total perturbation field requires hundreds of milliseconds to build up in the core of the device because of surrounding conducting structures, as can be observed in Fig.3. The upper panel shows the current flowing in one of the coils, while the lower one an estimate of the $n=1$ field component evolution inside the vessel as measured by large saddle coils located in the high field region (SAT coils). The current is turned on at $t=0 \mathrm{~s}$, but only after $\sim 300 \mathrm{~ms}$ the field in the plasma reaches its maximum value; even to reach half of the maximum about $150 \mathrm{~ms}$ are required. These times are comparable with the RE beam lifetime; this is why perturbations have been initially applied from $0.5 \mathrm{~s}$ before the disruption. Some experiments with the RMPs applied from a time following the disruptions have been also executed and are reported in Section VIII. Here and in Sections III-VII only discharges with perturbations pre-existing the disruption are considered. These experiments are still of general relevance, since in large machines operated at high magnetic field like ITER, a perturbation preceding the disruption might be useful to prevent the formation of huge RE beams difficult to mitigate with standard methods. Clearly, such an approach requires disruption prediction methods currently in development and investigation [42-45].

A differential phasing scan between the top and bottom coil set was carried out using the same ramp-up and gas injection scenario; the results for the RE current and HXR radiation are reported 


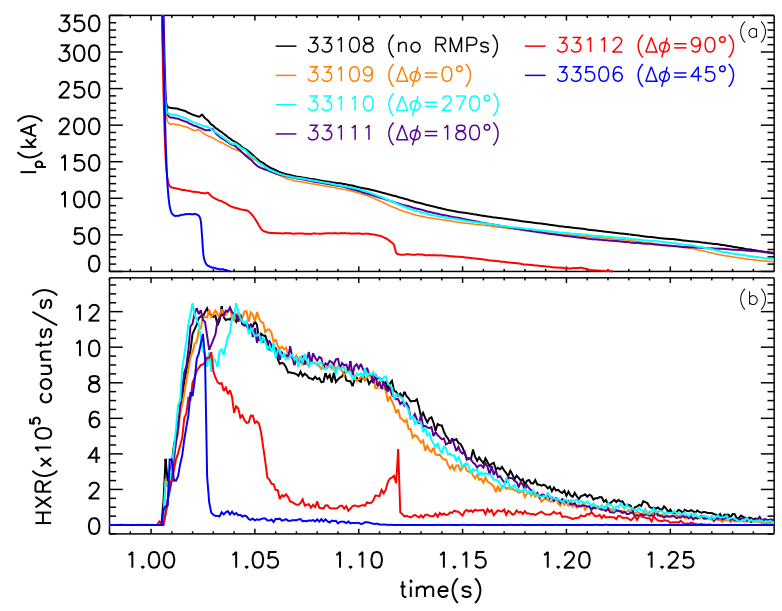

FIG. 4: (a) Plasma current during the post disruption phases for different values of $\Delta \phi$ and (b) corresponding HXR emission from $\operatorname{LaBr}_{3}(\mathrm{Ce})$ scintillator.

in Fig.4. Panel (a) shows that both the initial runaway current and the beam duration in the post-disruption phase are almost halved with respect to the unmitigated RE discharge without external field application when $\Delta \phi=45^{\circ}, 90^{\circ}$. Similarly, the HXR signal decreases by a factor of $\sim 2-3$ in less than $70 \mathrm{~ms}$, and remains close to zero afterwards. This phenomenology is not observed for other differential phases of the B-coils; no significant effect of the applied perturbation is visible for $\Delta \phi=0^{\circ}, 180^{\circ}$ and $270^{\circ}$, only in the first case a very slight reduction of the current with respect to the unperturbed current waveform occurs but without any important decrease of the HXR emission and within the statistical variation of standard unperturbed discharges. On the other side, the discharge reported in this plot with $\Delta \phi=90^{\circ}$ is characterized by a reduction of the RE current $(\sim-40 \%)$ and HXR emission $(\sim-60 \%)$. This behaviour is even more pronounced for $\Delta \phi=45^{\circ}$, where the runaway beam initial current is much lower than in the other discharges $(\sim 70$ $\mathrm{kA})$. It is worth to note the presence of sudden drops in the current waveforms for $\Delta \phi=45^{\circ}, 90^{\circ}$ correlated with spikes in the HXR signal, a signature of abrupt RE losses.

In the next three sections the main features of shots with RMPs will be discussed with respect to disruption dynamic, thermal quantities and hard-X-ray radiation.

\section{EFFECT OF RMPS ON DISRUPTION DYNAMICS}

The presence of an external $n=1$ field is found to be associated to a partial reduction of the time interval between the thermal and current quench phase in the disruption evolution. A 


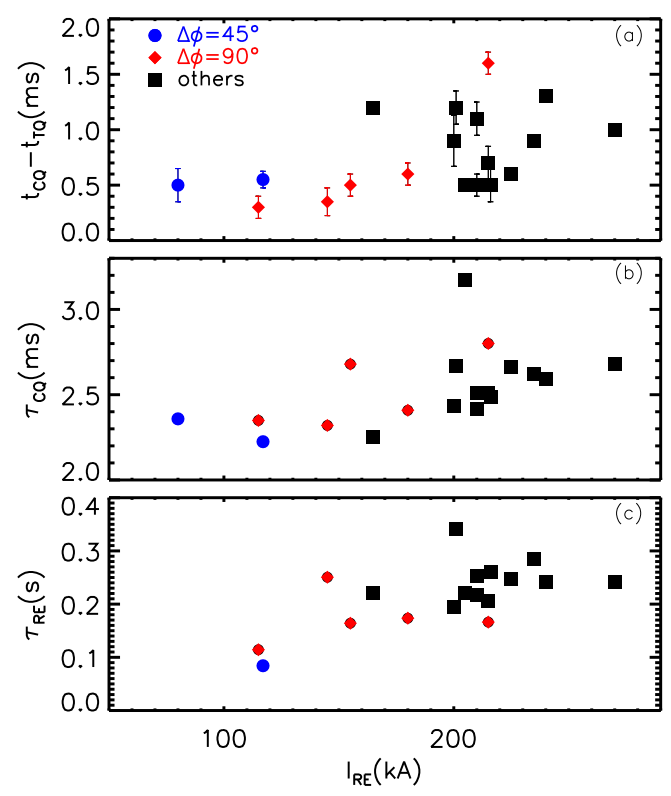

FIG. 5: Time interval between current and thermal quench (a) and current quench (b) and runaway beam (c) decay time as function of the final post-disruption RE current.

systematic evaluation of the thermal quench time $\left(t_{T Q}\right)$ has been performed using the soft-X-ray radiation from a central chord and has been defined as the time at which this signal exceeds a fixed reference threshold. Similarly, the time of the current quench $\left(t_{C Q}\right)$ corresponds to the maximum of the plasma current before the fall down. The time interval $\Delta t=t_{C Q}-t_{T Q}$ is reported in panel (a) of Fig.4 as function of the initial post disruption current $I_{R E}$. The blue-circles refer to shots with $\Delta \phi=45^{\circ}$ RMP while the red-diamonds to $90^{\circ}$; in black-squares other discharges without RMPs or with $\Delta \phi \neq 45^{\circ}, 90^{\circ}$ are reported. These data show that RMPs having the strongest impact on the final RE current/energy are characterized by a lower $\Delta t$ (generally $\lesssim 0.6 m s$ ) i.e. by an early current quench - which suggests a faster loss of the thermal content during the disruption.

A similar behavior is found for the evolution of the current quench phase and of the RE beam decay rate. For this analysis the $I_{p}$ current waveform during the quench phase, between the maximum value and the beginning of the RE plateau, has been interpolated by an exponential fit:

$$
\frac{I_{p}}{I_{C Q}}=e^{-\frac{t-t_{C Q}}{\tau_{C Q}}}
$$

where $I_{C Q}$ is the current at $t=t_{C Q}$ and $\tau_{C Q}$ defines a characteristic current quench time which is inversely proportional to the $I_{p}$ decay rate. Lower $\tau_{C Q}$ are thus associated to faster decrease of the plasma current after the disruption. As reported in Fig.5-(b), those shots which significantly 
reduce the initial $\mathrm{RE}$ beam current generally have $\tau_{C Q}<2.3 \mathrm{~ms}$ while most of the others range between this value up to $3.2 \mathrm{~ms}$. Both the shots with $\Delta \phi=45^{\circ}$ (blue circles) are characterized by values of $\tau_{C Q}$ among the lowest ones. For shots with $\Delta \phi=90^{\circ}$ (red diamonds) the dispersion is greater but still roughly scale with the final runaway beam current.

The characteristic runaway beam current decay time $\tau_{R E}$ has been estimated by a lineat fit:

$$
\frac{I_{p}}{I_{R E}}=1-\frac{t-t_{R E}}{\tau_{R E}}
$$

where $t_{R E}$ is the time at which the RE beam plateau begins and, as usual, $I_{R E}$ is the corresponding current. The results are reported in Fig.5-(c) with $\tau_{R E}$ vs $I_{R E}$. Most of the shots with the most efficient perturbations $\left(\Delta \phi=45^{\circ}, 90^{\circ}\right.$, blue circles and red diamonds) are characterized by significant reduced values of $\tau_{R E}$, lower than $0.2 s$. All the RE beams in shots without RMPs or with $\Delta \phi \neq 45^{\circ}, 90^{\circ}$, on the contrary, have a slower decay with $\tau_{R E}$ between $0.2 \mathrm{~s}$ and $0.4 \mathrm{~s}$. It is worth noting that one of the shot with $\Delta \phi=45^{\circ}$ is not reported in this plot since it was affected by a fast loss of position control.

These facts, in particular the reduced time interval between thermal and current quench and the different $\mathrm{CQ}$ decay rate for most efficient perturbations, suggest a general worsening of the confinement properties when RMPs with $\Delta \phi=45^{\circ}, 90^{\circ}$ are applied. Such a behavior might be explained in terms of an enhanced MHD activity leading to a faster degradation of the thermal confinement, as also confirmed by the electron temperature decrease reported in the next section, and thus to a sudden increase of the plasma resistivity. This might also affect the primary generation of runaway electrons, thus reducing the initial seed available, and/or the efficacy in the avalanche mechanisms. Also in past experiments in DIII-D a similar phenomenology was observed [46] when $n=3$ perturbations were applied before the disruption; there, in particular, discharges with RMPs application were characterized by current quench decay rates among the highest ones. In DIII-D too, these facts have been interpreted as a general worsening of the confinement properties due to the applied perturbations.

\section{IMPACT OF RMPS ON THERMAL QUANTITIES}

The application of perturbations is correlated to a different behavior of the main thermal quantities, like electron density and temperature, with respect to unperturbed shots. Fig.6 shows the density averaged in the time interval $0.9-1.0 \mathrm{~s}$ for an interferometer chord passing through the core of the plasma (panel (a)) and for an other one closer to the edge region (panel(b)) as function of 


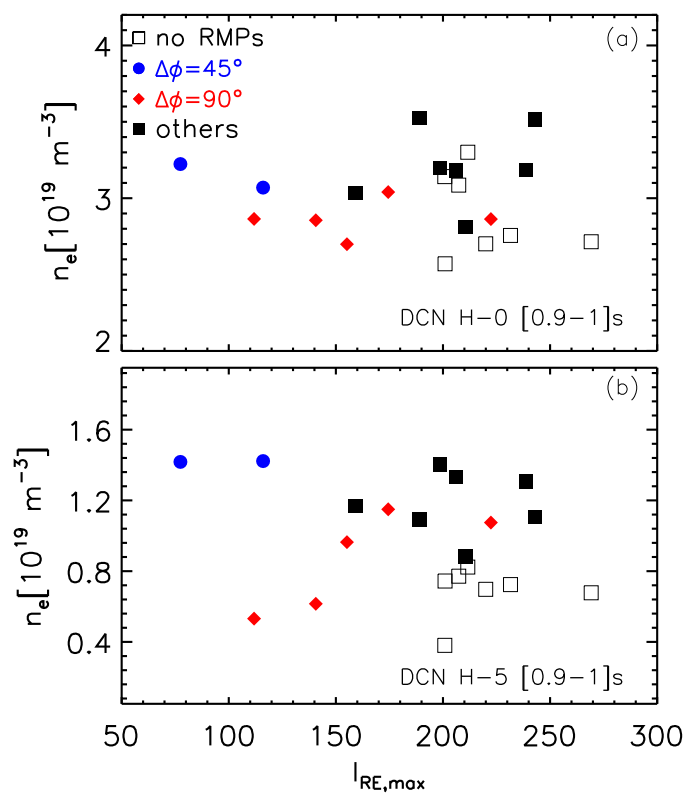

FIG. 6: (a) Core (H-1 chord) and (b) edge (H-5 chord) electron density before the disruption (averaged between $0.9-1 s$ ) as function of the post-disruption runaway current.

the final post-disruption runaway current $I_{R E}$. A clear difference between shots with $\Delta \phi=45^{\circ}, 90^{\circ}$ and other cases is not visible and the density spans between 2.5 and $3.5 \cdot 10^{19} \mathrm{~m}^{-3}$ in the core and between 0.4 and $1.6 \cdot 10^{19} \mathrm{~m}^{-3}$ at the edge. In this latter case the discharges with $\Delta \phi=45^{\circ}$ perturbations (blue circles) are among those with higher density but these values are also common to other shots which do not affect significantly the final runaway current. Moreover, when $\Delta \phi=90^{\circ}$, $I_{R E}$ decreases at larger densities. It is worth noting that most of the shots with perturbations are characterized by an increased density at the edge with respect to the average of standard discharges (empty squares). These plots suggest that the presence of perturbations might be correlated to an enhanced plasma-wall interaction but that is not responsible of the mitigation effect on runaways. Indeed, panel (b) of Fig.6 clearly state that the perturbation effect on density is not correlated with their efficiency in reducing the final runaway beam current. For instance few of the shots with $\Delta \phi \neq 45^{\circ}, 90^{\circ}$ (black full squares) and those with $\Delta \phi=45^{\circ}$ are characterized by a similar density but only the latter successfully reduce $I_{R E}$. On the contrary, there are shots with $\Delta \phi=90^{\circ}$ significantly affecting the runaway current with density closer to those typical of standard shots. Thus we can conclude that the mitigation effect of RMPs on runaways is not a mere consequence of different density regimes induced by the applied perturbations before the disruption.

The electron temperature profile measured by ECE diagnostic follows the same evolution during 


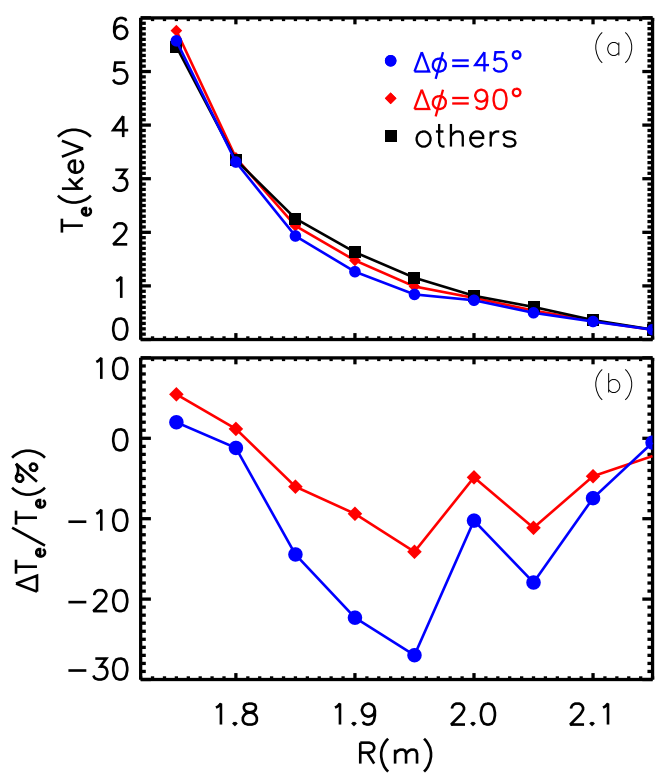

FIG. 7: Averaged electron temperature profiles zoomed in the mid/edge region of the plasma (a) and percentage variations with respect to unperturbed discharges (b) in the time interval $0.9-1 \mathrm{~s}$.

the time interval $0-0.9 s$ regardless of the RMPs application. Nevertheless, during the last $100 \mathrm{~ms}$ when ECRH is applied, the $T_{e}$ profile of discharges with $\Delta \phi=45^{\circ}$ are characterized by a region of lower temperature. This can be appreciated in Fig.7-(a) reporting $T_{e}(R)$ profiles averaged both in time (during $0.9-1 \mathrm{~s}$ ) and over more shots in the mid/edge region of the plasma. The black (squares) line refers to unperturbed discharges while the blue line (circles) to those with $\Delta \phi=45^{\circ}$. While the profiles are very similar in the edge region $(R>2 \mathrm{~m})$, between $R=1.8 \mathrm{~m}$ and $R=2.0 \mathrm{~m}$ an important difference appears. Something of analogous, but of smaller extent, is observed also when $\Delta \phi=90^{\circ}$ (red line or diamonds). In panel (b) of the same figures the percentage deviation of temperature $\left(\Delta T_{e} / T_{e}\right)$ from the standard cases is plotted both for $\Delta \phi=90^{\circ}$ and $\Delta \phi=45^{\circ}$; while in the former case such a deviation is limited below the $\sim 10 \%$, for the latter it can increase up to $30 \%$ around $R=1.9 \mathrm{~m}$ corresponding to an absolute reduction in temperature also of $400 \mathrm{eV}$. Such a decrease of the electron temperature with respect to the unperturbed discharges could result in a lower hot tail RE seed current, which is exponentially dependent on the initial $T_{e}$ [47], and also in a higher resistivity which in turn might affect the primary RE generation. 


\section{EFFECT OF RMPS ON HXR ENERGY SPECTRUM.}

The hard-X-ray radiation emitted by the runaways has been detected by a $\operatorname{LaBr}_{3}(\mathrm{Ce})$ spectrometer with a counting rate capability up to $\mathrm{MHz}$ and high energy resolution $(3 \%$ at $667 \mathrm{keV})$ [48]. The detector measures gamma-rays, emitted at about 90 degrees with respect to the magnetic field in the core, along a radial line of sight passing through the plasma center. With the present settings of the photomultiplier, the maximum gamma-ray energy detectable is approximately 20 $\mathrm{MeV}$. Four examples of HXR spectra, normalized to the total number of events, are reported in Fig. 8 in the time interval between 1 and $1.05 \mathrm{~s}$ (just after the disruption): the black curve refers to a standard discharge (no RMP applied), while the orange, red and blue ones to perturbed shots with $\Delta \phi=0^{\circ}, 45^{\circ}, 90^{\circ}$ phasing respectively. By analyzing the slope of these distributions it is found that the $45^{\circ}$ trace falls off clearly faster in the range $300-800 \mathrm{keV}$, with the exception of a quasi constant region between 400 and $500 \mathrm{keV}$ which is of comparable shape also in the $90^{\circ}$ discharge. On the other side, no significant differences in the HXR spectrum between the unperturbed and the $\Delta \phi=0^{\circ}$ shot are observed, both display an increased high energy content with respect to the other two discharges. The variations in the HXR spectrum qualitatively reflect the changes in the RE energy distribution even if a more detailed determination requires dedicated methods [49] and modeling activity which is currently in progress. However, most of the gamma-rays that are born in the plasma experience negligible scattering in their path from their birth position to the detector. Some, however, can change their energy if they interact with some structures once escaped from the plasma. This second process can slightly alter the shape of the spectrum with respect to the ideal case where gamma-rays do not scatter on materials as they travel from the plasma to the detector. The contribution of this scattering of radiation in the pulse height spectrum is the same in the RMP and no-RMP case, as the viewing geometry does not change between the two cases, and cannot explain the different shapes obtained, unless the source (i.e. the energy distribution of the runaway electrons) has changed.

A more detailed analysis to quantify the effect of perturbations in reducing the high energy components of HXR distributions has been performed and reported in Fig.9. For each spectrum the average slope on the right of the HXR peak has been evaluated by a power fit $\left(\propto E^{-\alpha}\right)$. In particular the coefficient $\alpha$ has been computed for the whole database of shots considered in this paper, in two different energy ranges (lower: $300-800 \mathrm{keV}$, higher : $>800 \mathrm{keV}$ ) and in the following time intervals: $1-1.05 s, 1.05-1.1,1.1-1.15 s$. The results have been plotted both as function of the averaged RE current in the selected temporal intervals (a)-(b) and of the phasing of the 


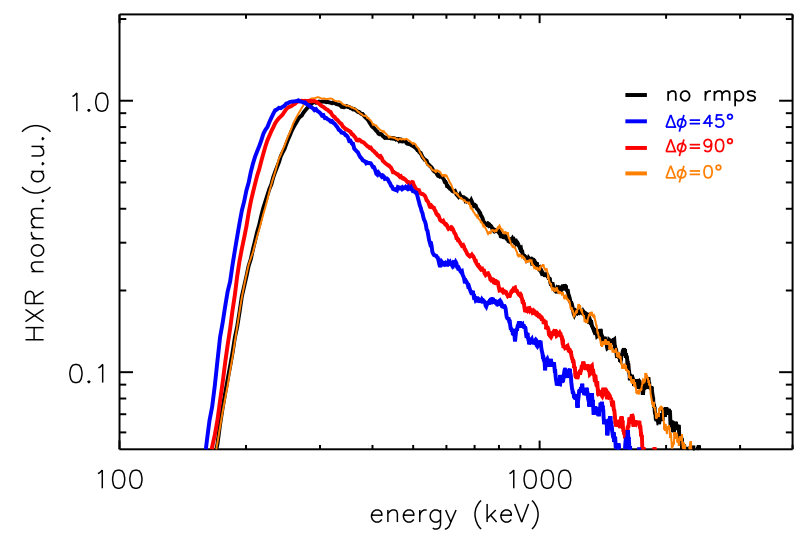

FIG. 8: (a): HXR energy spectrum from $\mathrm{LaBr}_{3}(\mathrm{Ce})$ scintillator diagnostic for standard shot (black,\#33505) and for three with RMPs applied but with different $\Delta \phi\left(45^{\circ}\right.$-blue curve $\# 33506,90^{\circ}$ - red curve \#33112, $0^{\circ}$ - orange curve \#33109).
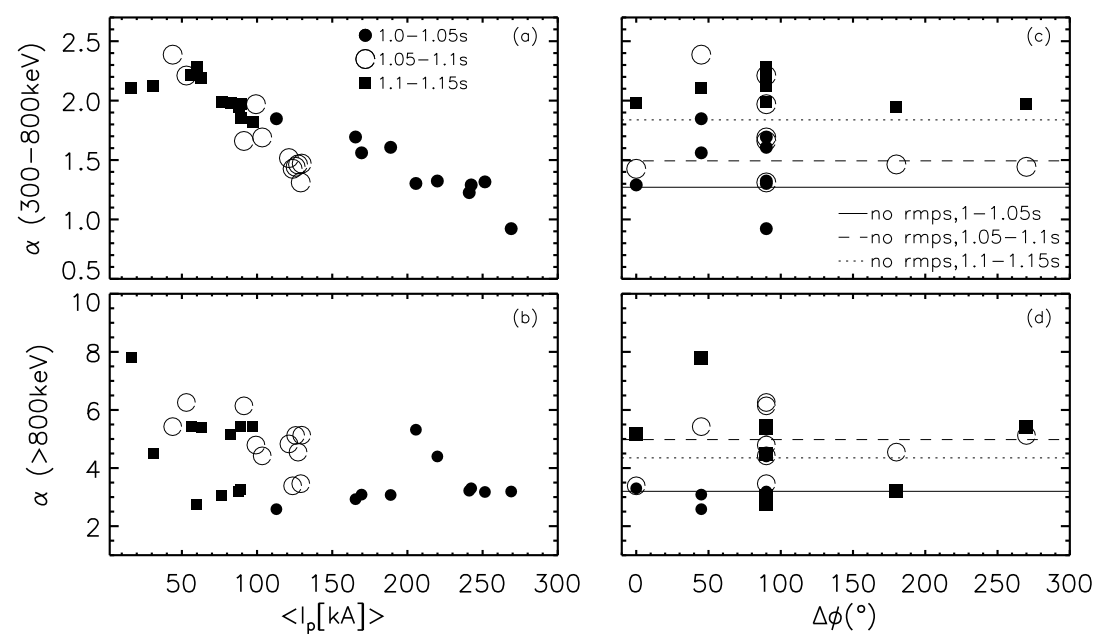

FIG. 9: (a): Slope of HXR energy spectrum from in the energy range $300-800 \mathrm{keV}$ and (b) $>800 \mathrm{keV}$ as function of the average current in different time intervals. (c)-(d): Same data but plotted versus the B-coil phasing.

applied perturbations. Panel (a) clearly shows that at lower energies, below $800 \mathrm{keV}$, the slope of the spectra scale with the current of the RE beam decreasing from $\sim 2-2.5$ at $I_{R E} \sim 50 \mathrm{kA}$ to $\sim 1$ more typical of initial post-disruption current $\left(I_{R E} \sim 200 \mathrm{kA}\right)$. The same behavior is not observed for high energies in panel (b) where $\alpha$ range between 2 and 8 but without a clear trend; nevertheless higher values of $\alpha$, at least greater than 4.5 are found only at lower $\left\langle I_{R E}\right\rangle$, thus in 
a later phase of the beam evolution or when most efficient RMPs are applied.

The $\alpha$ evolution for different phasing of the perturbations is displayed in panel (c) and (d). The horizontal lines correspond to the average $\alpha$ for shots without perturbations (continuous for $1-1.05 s$, dashed for $1.05-1.1 s$ and dotted for $1.1-1.15 s$ ). At lower energies (panel (c)) an increase of $\alpha$ in time occurs for all the shots considered but the highest increase is relative to those with $\Delta \phi=45^{\circ}$. A large dispersion appears for $\Delta \phi=90^{\circ}$ characterized by values also below the ones of unperturbed shots. At higher energy (panel (d)), as for the RE current, a clear difference between shots with perturbations and without is difficult to be appreciated; the only exception is again represented by one discharge with $\Delta \phi=45^{\circ}$, that, in the third time interval considered $(1.1-1.15 s)$, is characterized by a very high $\alpha$, close to 8 .

More modeling is of course required to draw more direct information on the RE physics from the HXR measurements; nevertheless, the data reported here show that most efficient RMPs act in order to reduce the post-disruption current which, in turn, is associated to steeper HXR spectra but with a strength depending on the energy ranges considered.

\section{ROLE OF PLASMA RESPONSE IN RE MITIGATION}

The distinctive behavior of the phase $\Delta \phi=45^{\circ}$ with respect to others, as analyzed in the previous sections in relation to several quantities, will be discussed in this Section. The poloidal spectrum of the applied 3D fields has been evaluated in vacuum approximation at the time $t=0.98 \mathrm{~s}$, just before the disruption event, for more differential phases of the B-coils. Panels on the top of Fig.10 show the perturbed field as function of the poloidal wave number $m$ and of the normalized poloidal flux coordinate $\left(\rho_{\text {pol }}\right)$ for $\Delta \phi=0,45^{\circ}, 90^{\circ}, 135^{\circ}, 270^{\circ}, 315^{\circ}$, respectively. The safety factor profile $\left(q=r B_{T} / B_{P} R_{0}\right.$ with $B_{T}, B_{P}$ the toroidal and poloidal field respectively and $r, R_{0}$ the minor and major radius of the plasma) is plotted with a white dashed line. The $\Delta \phi=45^{\circ}$ case shows that the maximum perturbed field occurs in the edge region and between the non-resonant component $m=5$ and $m=6$; the resonant position with $q=4$ on the other hand lies in a region of low field $(<1.5 \mathrm{G})$. By increasing the B-coil phase difference, such a maximum is continuously shifted to lower values of $m$ and at $\Delta \phi=315^{\circ}$ intersects the $q$ profile and in particular the rational surfaces $q=3$ and $q=4$. As the parameter to quantify the variation of the RMP amplitude $b_{r}$ with $\Delta \phi$, the value of the $m=4$ perturbed field component at the resonance $q=4$ was evaluated and shown in Fig.11(a) with a black-dashed line. As clear from this plot, the $n=1$ radial field resonant with $q=4$ (in the vacuum approximation) is maximum when 

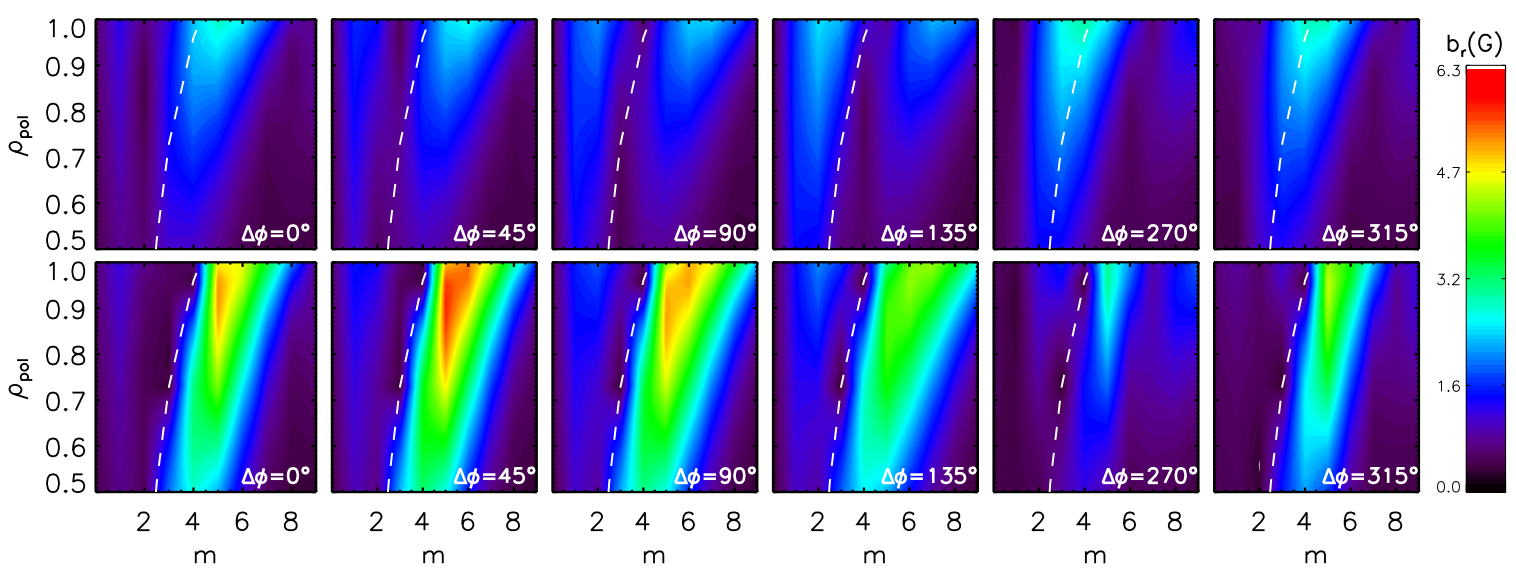

$24 \quad 6 \quad 8$

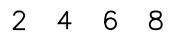

$\mathrm{m}$

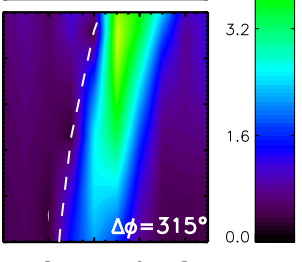

2468

$\mathrm{m}$

FIG. 10: Contour of perturbed field as function of the $m$ component and normalized poloidal flux in vacuum approximation (top panels) and including the plasma response as computed by the code MARS-F (bottom panels) for the shot \#33113 at 0.98 s.

$\Delta \phi \sim 315^{\circ}$ with a minimum value close to zero around $\Delta \phi \sim 100^{\circ}$; a similar trend is found also for the $m=3$ mode estimated at $q=3$ position (not shown in the figure for the sake of clarity). Such a dependence cannot explain the experimental data described in the previous sections summarized in Fig.11-(b)-(c) showing the initial RE beam post-disruption current and its lifetime (the latter evaluated as the time from the current quench until $I_{R E}$ drops below 10kA). Here, each point corresponds to a different discharge and the dashed line to the average post-disruption current for shots where RMPs are not applied. As already observed below, in the two shots with $\Delta \phi=45^{\circ}$ the initial runaway electron beam current is reduced by more than a factor of 2 . A similar effect is found when $\Delta \phi=90^{\circ}$. No RE mitigation is observed for $\Delta \phi \sim 315^{\circ}$ where RMPs give the largest vacuum contribution to the rational surface $q=4$ (or $q=3$ ).

The code MARS-F [50, 51], which solves the single-fluid linearly perturbed MHD equations in full toroidal geometry, has been used to calculate the poloidal spectrum including the plasma response to RMPs. With respect to standard vacuum simulations, MARS-F takes into account the plasma resistivity, important in those processes involved in the magnetic islands formation, the plasma flow, which normally tends to shield the external field from penetrating through the plasma and finally the coil geometry, since the spectrum of the resonant harmonics depends largely on the plasma shape. The equilibrium is provided by the code CLISTE and the kinetic quantities such as electron temperature and density profiles are provided by Integrated Data Analysis (IDA) [52] of diagnostics. In the following simulations a toroidal rotation of $\omega / \omega_{A}=5 \cdot 10^{-3}$ is assumed $\left(\omega_{A}\right.$ 
is the Alfvén frequency for these plasmas). Bottom panels in Fig.10 show the results obtained relative to an equilibrium preceding the disruption (reference shot: $33113, t=0.98 \mathrm{~s}$, the same used for the vacuum field reconstruction). At all values of the differential phase considered, the plasma response reduces the amplitude of resonant harmonics at the corresponding rational surfaces compared with the vacuum field; on the other hand the kink relative to the components $m=5,6$ for $\Delta \phi=45^{\circ}$ is enhanced by more than a factor 3 . The same analysis has been performed for more $\Delta \phi$ and toroidal rotation values. As for the vacuum field, the $m=4$ resonant component at $q=4$ is shown in Fig.11-(a) with red squares; its amplitude is reduced by a factor $\sim 8$ with respect to the vacuum approximation. The differential phase relative to the maximum is shifted by $\sim 45^{\circ}-60^{\circ}$ in the direction of increasing $\Delta \phi$ values, thus closer to the B-coil differential phase experimentally more successful in reducing the RE beam current. The same panel reports also the average amplitude of the non resonant mode $m=5$ (blue-diamonds curve) in the edge region $\left(\rho_{\text {pol }}>0.8\right)$. A clear maximum can be observed at $\Delta \phi \sim 45^{\circ}-90^{\circ}$ with an absolute value higher than $5 \mathrm{G}$; the RMP configuration for which runaway electrons are best mitigated is thus related to the largest edge kink response. A similar behavior was found for the B-coil configuration most efficient in suppressing edge localized modes (ELMs) [53, 54], where the maximum field evaluated including the plasma response is offset $\sim 60^{\circ}$ from the one in vacuum approximation [55].

The results presented here, in particular the dependence of the maximum $b_{r}$ vs $\Delta \phi$, do not vary significantly with the toroidal rotation as can be observed in Fig.12. Panel (a) shows the average $m=5$ component at the edge for $\omega / \omega_{A}$ increasing from $10^{-3}$ to $5 \cdot 10^{-2}$; the curves are very close one each other and present the maximum value around $45^{\circ}$. The same analysis but for the $(4,1)$ resonance is reported in panel (b); at larger rotation velocity, the plasma is more shielded from the penetration of external field and the resonant component is reduced. The phasing corresponding to the maximum perturbed field at the $(4,1)$ resonance increases with rotation from $0^{\circ}-45^{\circ}$ to $45^{\circ}-90^{\circ}$. An estimate of the experimental toroidal flow can be inferred from the rotation frequency of the inner $(1,1)$ mode when present and is of the order of $\sim 5$ $\mathrm{kHz}\left(\omega / \omega_{A} \sim 2-3 \cdot 10^{-3}\right)$; thus the experimental data analyzed in this paper correspond to an intermediate position between the black (circles) and red (squared) curves.

The total perturbed field (vacuum + plasma response) of the resonant field components might be relevant in the formation of magnetic islands (e.g. harmonics $(4,1)$ and $(3,1)$ ) and, potentially, can be responsible for field line ergodization. But also the non-resonant part (kink amplification), that does not necessarily ergodize fields, further contributes to RE suppression (by coupling to resonant spectrum or direct orbit modification). In this sense, the combined 


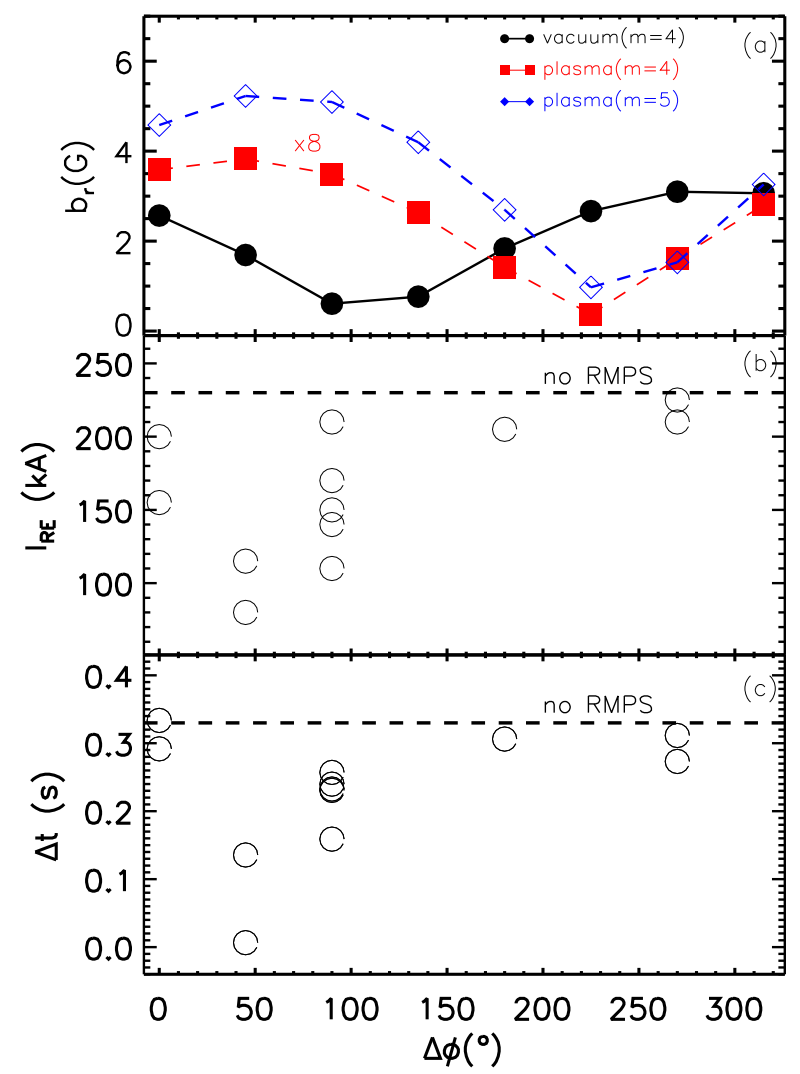

FIG. 11: (a) Perturbed field amplitude at the resonance $q=4$ and of the kink mode $m=5$ in the edge region, (b) post-disruption RE current and (c) lifetime. The dotted line corresponds to the average RE current in standard discharges.

results reported in Figure 10-11 point to two mechanisms both occurring at the same favorable coil phasing which could affect the primary generated runaway electrons, thus reducing the initial seed, or those produced in the avalanche process. Nevertheless, a deeper understanding of these issues would require a detailed analysis directly by modeling the RE trajectories in these 3D fields [56] and/or an investigation with a two-fluid approach [57], considering also non-linear effects in the plasma response to RMPs. When two-fluid terms are included in the response calculations, the ion and electron rotation velocities are no longer the same; in particular the electron velocity results to be the relevant quantity controlling the field penetration in the core of the plasma at the mode-rational surface. Conversely, the excitation of edge modes is mainly correlated with the ion velocity [58]. Such an analysis might contribute to determine the necessary and sufficient conditions for the development of magnetic islands and of stochasticity. 


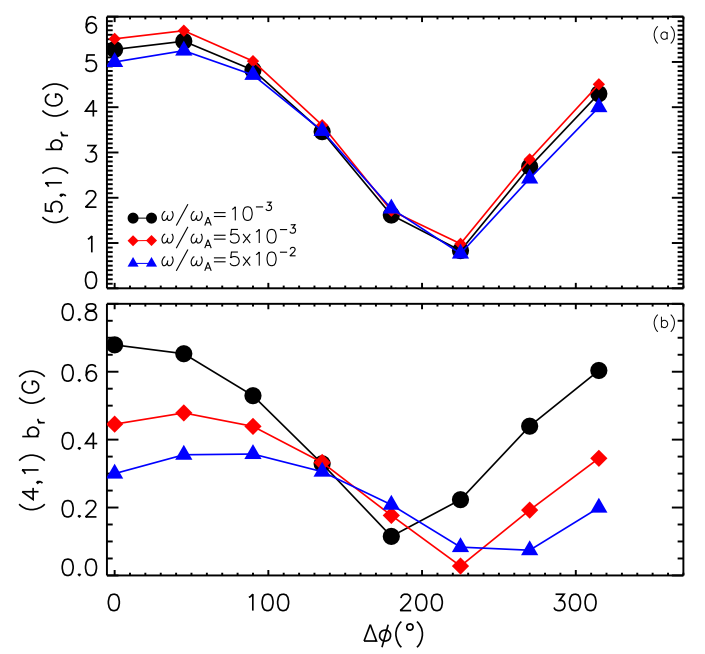

FIG. 12: (a) Perturbed field amplitude for the kink mode $n=5$ in the edge region (a), and at the resonance position $(4,1)(b)$ at different plasma rotations.

\section{RMPS APPLIED WITH LOWER AMPLITUDE AND IN THE POST-DISRUPTION PHASE}

The impact of different amplitude of RMPs applied before the disruption with the most efficient phasing $\left(\Delta \phi=45^{\circ}\right)$ has also been investigated. This has been done both by decreasing the current in the B-coils or keeping the maximum value available and varying the time at which they are switched on; indeed in this latter case, due to the slow diffusion time in the plasma, the perturbed field at the disruption is only a fraction of its final maximum value. Examples are reported in Fig.13-(a) showing one shot with $I_{B}=0.8 \mathrm{kA}$ from $t=0.5 \mathrm{~s}$ (blue solid) and two with $I_{B}=1 \mathrm{kA}$ from $t=0.8 \mathrm{~s}$ (solid orange and red lines); as references, also an unperturbed discharge is reported (black) and one with $I_{B}=1 \mathrm{kA}$ from $t=0.5 \mathrm{~s}$ (dashed blue line). Despite slightly lower, the post-disruption RE current for shots with RMPs of reduced amplitude is very close to the one of the discharge without perturbations. Nevertheless the solid blue line $\left(I_{B}=0.8 \mathrm{kA}\right.$ from $t=0.5 \mathrm{~s})$ is characterized by a faster decay of the $\mathrm{RE}$ beam with respect to the others and is more similar to that of the dashed line (full amplitude RMPs from $t=0.5 \mathrm{~s}$ ). These results highlight that a $\mathrm{B}$-coil current of $1 \mathrm{kA}$ is probably close to the minimum value required to see any effect on the runaways. Such a finding is still in agreement with the plasma response modeling presented 


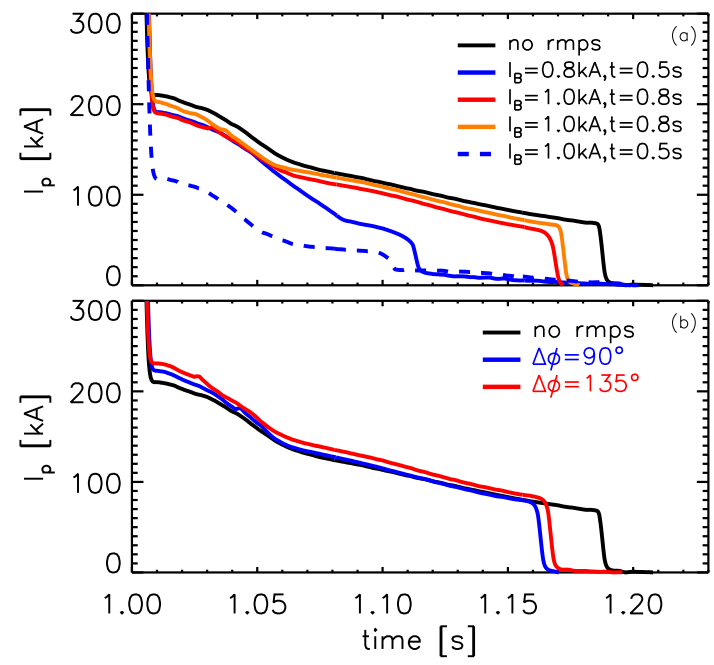

FIG. 13: (a) $\Delta \phi=45^{\circ}$ RMPs with different time of application and amplitude (black-\#34150 reference shot without RMPs, blue solid-\#34083, red-\#34145 orange-\#34146, blue-dashed: \#33706- $I_{B}=1 \mathrm{kA}$ from $t=1 \mathrm{~s}$ ); (b) Examples of discharges with RMPs applied from $t=1 \mathrm{~s}$ (black-\#34150, blue-\#34143, red\#34144);

in the previous section as illustrated in Fig.14. Panel(a) shows again the radial field associated to the $m=5$ component computed by MARS-F as function of the phasing; the maximum value at $\Delta \phi=45^{\circ}$ is of $\sim 5.5 \mathrm{G}$. Shots with RMP of reduced amplitude or with a delay of their application correspond to a different radial field associated. In the examples reported in Fig.13-(b), the blue curve and the orange/red ones correspond to an effective field of $72 \%$ and $65 \%$ with respect to the maximum available; in panel (a) of Fig.14 the corresponding $b_{r}$ values are indicated with dotted lines. Panel (b) of the same figure shows the post-disruption RE current as function of $\Delta \phi$ for discharges with the maximum (empty circles) and reduced (full symbols) RMPs amplitude. The latter data, as highlighted by the vertical dotted lines, are still in the trend traced out by the empty symbols; the reduced amplitude of the applied perturbation is equivalent to a radial field for the $m=5$ kink mode generated by a different phasing (between $90^{\circ}$ and $180^{\circ}$ in these cases) with $I_{B}=1 \mathrm{kA}$ and starting time $t=0.5 \mathrm{~s}$. Panel (c) shows a combination of the other two panels, $I_{R E}$ vs the $m=5$ radial field applied by the B-coils as evaluated by MARS-F; within some dispersion a decreasing trend is observed. Such a plot highlights that only a very narrow region in $b_{r}$ is available for $\mathrm{RE}$ mitigation and confirms that $I_{B}=1 \mathrm{kA}$ is close to the minimum threshold required to successfully mitigate the runaways. The variability observed in those cases 

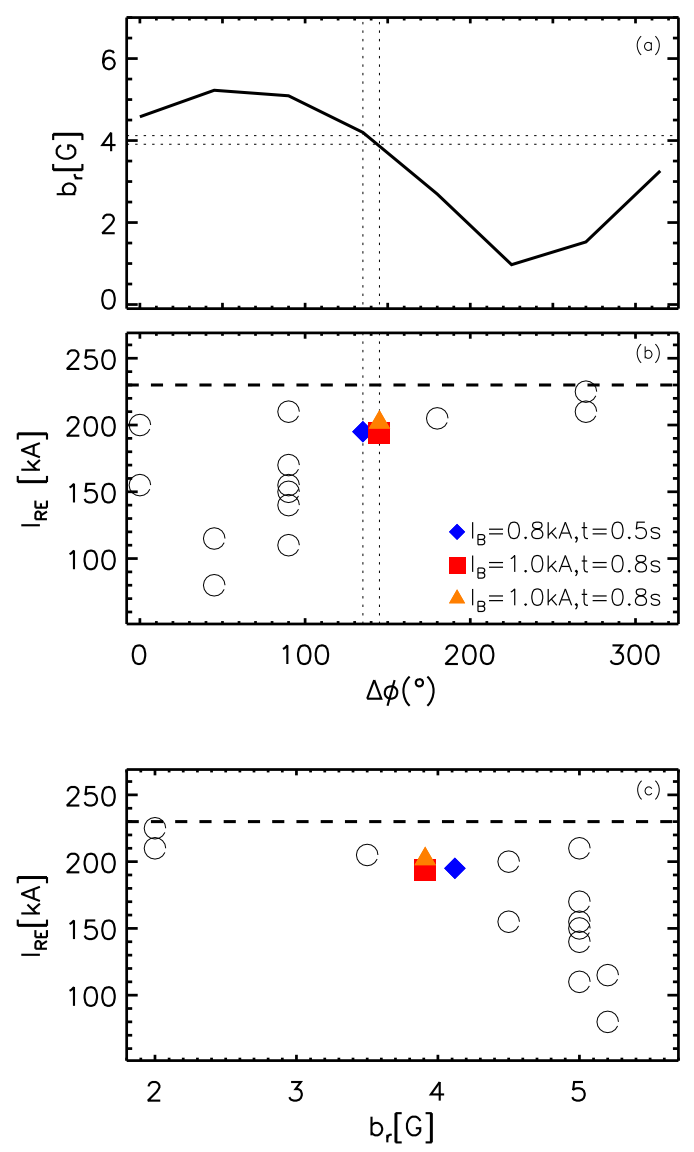

FIG. 14: (a) $m=5$ perturbed field evaluated by MARS-F and (b) post-disruption current vs $\Delta \phi$ with included also the shots with different RMP amplitude and timing (square,triangle, diamond). (c) Postdisruption current vs the corresponding $m=5$ perturbed field computed by MARS-F.

with $\Delta \phi=90^{\circ}$ may be due to the highly non linear dependence and to tiny variations of plasma response when the perturbation amplitude is around the threshold. A larger current in the B-coil might further reduce the runaway current and potentially increase the $\Delta \phi$ range useful in $\mathrm{RE}$ mitigation.

The results and considerations reported up to here refer to experiments with perturbations applied from a time preceding the disruptions, when plasmas are characterized by $\beta$ values significantly greater than zero. On the contrary, post-disruption RE beams are generally almost cold plasmas with reduced minor and major radius as was shown in Fig.2. In these cases, a plasma response mechanism can be neglected and a vacuum approximation - while analyzing the effects of applied perturbations on runaways - is still adequate. In order to verify if RE mitigation occurs also with RMPs applied only once the RE beam is fully developed, few shots have been executed 
turning on the B-coils at $t=1 \mathrm{~s}$. By this choice RMPs do not interfere with the disruption dynamic since at least $200-300 \mathrm{~ms}$ are required for the fields to diffuse in the plasma core, as shown in Section III. A preliminary estimate of the phasing which could mostly affect the RE beam in the post-disruption phase is more difficult since the equilibrium is not stationary. Vacuum field reconstruction at several times show that, at least up to $1.2 \mathrm{~s}$, the differential phases between $50^{\circ}$ and $150^{\circ}$ maximize the RMPs components at the resonance positions closer to the edge. For this reason perturbations with $\Delta \phi$ equal to $90^{\circ}$ and $135^{\circ}$ from $t=1 s$ have been applied; these shots are reported in Fig.13-(b) together with an unperturbed discharge belonging to the same experimental session. The evolution of RE current is rather similar for the three shots; the small variation in the initial current is not to be correlated with the effect of perturbations since their amplitude at $t \simeq 1$ s inside the plasma is almost zero. Unfortunately, these shots are characterized by a fast loss of equilibrium between $1.15 \mathrm{~s}$ and $1.2 \mathrm{~s}$ thus much before the typical duration of the RE beam. On the other side, up to $t=1.2 \mathrm{~s}$ the applied fields have not still completely built up in the plasma so it is not straightforward to conclude from these experiments if RMPs can affect an already developed RE beam or totally exclude this possibility.

\section{CONCLUSIONS.}

The threat of post-disruption generated runaway electrons to in-vessel and plasma facing components in fusion devices requires the development of appropriate mitigation techniques. In ASDEX Upgrade a scenario dedicated to investigate methods which allow reducing the negative effects associated to runaway electrons has been established; a disruption is induced by argon puffing in a circular 0.8MA plasma followed by the generation of a runaway beam with an initial current of $200-300 \mathrm{kA}$ and a duration up to $0.5 \mathrm{~s}$. Resonant magnetic perturbations with toroidal number $n=1$ have been applied by the B-coils with the maximum current available $(1 \mathrm{kA})$ and turned on $0.5 \mathrm{~s}$ before the disruption. The poloidal spectrum of the perturbations can be varied by changing the differential phase $\Delta \phi$ between the currents in the upper and lower coils. Experiments show that only few values of $\Delta \phi$ can significantly reduce the post-disruption runaway current and correspond to poloidal spectra which maximize the plasma response to the RMPs, as computed by MARS-F numerical simulations. These performing perturbations mainly affect the pre-disruption phase of the discharge as indicated by the reduction of the electron temperature profile by about $\sim 30 \%$ in the region between $R=1.8 \mathrm{~m}$ and $R=2 \mathrm{~m}$. Such a fact indicates a general worsening 
of the confinement properties and an enhanced heat transport as also confirmed by the disruption dynamic, characterized by a lower time interval between the current and thermal quench phase for the most efficient RMPs. Also HXR measurements show a reduction of the high energy content (below $1 \mathrm{MeV}$ ) in those discharges where a significant mitigation of the $\mathrm{RE}$ current is obtained. Despite the detailed mechanisms involved in the RE mitigation when efficient RMPs are applied require further investigation and modeling, the findings described above might be explained in term of several causes like: (1) a reduction in the initial hot tail RE generation, which exponentially depends on the $T_{e}$ profile (2) a variation of the initial pre-disruption rotation profile which can affect field penetration and the thermal quench dynamic - and thus indirectly also the RE confinement (3) the formation of magnetic islands and ergodic regions (4) an effect on impurity transport during the disruption - which could interfere with the way the injected argon is mixed into the plasma.

Reduced amplitude RMPs, still applied from a time preceding the disruption, only marginally affect the initial runaway current; indeed the maximum current available for the B-coils in these experiments is found to be very close to the minimum threshold required to have any significant effect on the runaway beam. Perturbation applied after the disruption do not seem to affect the RE beam evolution but the recurring loss of RE beam position control in these experiments prevents to draw definitive conclusions on this issue, since the fields - due to the surrounding conductive structures - might require times longer than $200 \mathrm{~ms}$ to penetrate the plasma.

The results presented in this paper are not in contradiction with previous ones observed in other devices; on the contrary, they make us rather suppose that, in past experiments, the RMPs poloidal spectrum could not have been the most efficient one due to an unfavorable phasing or to the geometry of the coils. These findings, in synergy with standard mitigation methods, might be relevant for RE suppression in future fusion reactors.

Acknowledgments. A special thanks to R.Fischer for providing us the inputs data for MARS-F simulations. This work has been carried out within the framework of the EUROfusion Consortium and has received funding from the Euratom research and training programme 2014-2018 under grant agreement No 633053. The views and opinions expressed herein do not necessarily reflect those of the European Commission. Work supported by US DoE Office of Science under Contract DE-FG02- 95ER54309 and DE-FC02-04ER54698.

[1] The ITER physics basis. Nucl. Fusion 47, S1-S413 (2007).

[2] Hender T. C. et al. Nucl. Fusion 47, S128202 (2007) 
[3] E.C.Hollmann et al. Phys.Plasmas 22, 056108 (2015)

[4] M. Lehnen et al. JNM 46339 (2015)

[5] R.S.Granetz et al. Phys. Plasmas 21,072506 (2014)

[6] M.N.Rosenbluth and S.V.Putvinski, Nucl.Fusion 37, 1355 (1997)

[7] J. W. Connor and R. J. Hastie, Nucl. Fusion 15, 415424 (1975)

[8] R.Aymar, et al, Plasma Phys. Control. Fusion 44, 519 (2002)

[9] Hender T.C. et al. , The ITPA MHD, Disruption and Magnetic Control Topical Group 2007 Nucl. Fusion 47 S128

[10] A.H.Boozer Nucl. Fusion 57, 056018 (2017)

[11] G.Papp et al. Plasma Physics and Controlled Fusion, 54 (2012)

[12] M. Lehnen et al. Journal of Nuclear Materials 4633948 (2015)

[13] Eidietis N W et al Phys. Plasmas 19056109 (2012)

[14] B.Esposito et al Plasma Phys. Control. Fusion 59014044 (2017)

[15] Bakhtiari M. et al Nucl. Fusion 42, 1197 (2002)

[16] S. A. Bozhenkov et al Plasma Phys. Control. Fusion 50, 105007 (2008)

[17] C.Reux et al, Nucl. Fusion 50, 095006 (2010)

[18] Z. Y. Chen, Plasma Phys. Control. Fusion 55, 014046 (2017)

[19] G.Pautasso et al, Plasma Phys. Control. Fusion 59, 014046 (2017)

[20] M. Lehnen et al Nucl. Fusion 53 (9), 093007 (2013)

[21] Taylor P.L. et al Phys. Plasmas 6, 1872 (1999)

[22] N.Commaux et al Nucl. Fusion 56046007 (2016)

[23] G.M.Olynyk et al Nucl. Fusion 53092001 (2013)

[24] P.Helander et al Phys.Plasmas 7, 4106 (2000)

[25] R.W.Harvey et al, Phys. Plasmas 7, 4590 (2000)

[26] G.Papp et al. Journal of Plasma Physics 8147581050 (2015)

[27] K.Aleynikova et al., P. Plasma Phys. Rep. 42, 486 (2016)

[28] M.Lehnen et al, Phys.Rev.Lett. 100, 255003 (2008)

[29] K. Wongrach1 et al Nucl. Fusion 55, 053008 (2015)

[30] Hollmann E.M. et al Phys. Plasmas 17056117 (2010)

[31] R.Yoshino et al, Nucl.Fusion 40, 1293 (2000)

[32] M.Gobbin et al. Nucl. Fusion 57, 016014 (2017)

[33] T.Evans et al., Phys. Rev. Lett. 92, 235003 (2004)

[34] M.Gobbin et al. submitted to Phys. Rev. Lett.

[35] A.Kallebanch et al. to be published on Nuclear Fusion (IAEA 2016)

[36] W.Suttrop et al.. Phys. Rev. Lett. 106225004 (2011)

[37] Y.Q.Liu et al. Nucl. Fusion 51 (2011)

[38] G.Papp et al. to be published in Nuclear Fusion (IAEA 2016) 
[39] M.Nocente et al Rev. Sci. Instrum. 81, 10D321 (2010)

[40] M.Nocente et al IEEE Trans. Nucl. Sci. 601408 (2013)

[41] P. J. McCarthy and ASDEX Upgrade Team, Plasma Phys. Control. Fusion 54, 015010 (2012).

[42] G. A. Rattá, et al., Nuclear Fusion 50025005 (2010)

[43] G.Pautasso et al., Nuclear Fusion 51103009 (2011).

[44] S. Dormido-Canto et al., Nuclear Fusion 53113001 (2013).

[45] P. De Vries et al Nuclear Fusion 562 (2016)

[46] N.Commaux et al. Nucl. Fusion 51, 103001 (2011)

[47] H. M. Smith and E. Verwichte Phys. Plasmas 15, 072502 (2008)

[48] M. Nocente et al. Nucl. Fusion 57076016 (2017)

[49] A.E. Shevelev et al Nucl. Fusion 53 (2013) 123004

[50] Y.Q.Liu et al. Phys. Plasmas 73681 (2000)

[51] Y.Q.Liu et al. Phys. Plasmas 17122502 (2010)

[52] R.Fischer et al. Fusion Sci. Technol.58 675 (2010)

[53] F.Orain et al. Nucl. Fusion 57022013 (2017)

[54] L.Li et al. Nucl. Fusion 56, 126007 (2016)

[55] D.A.Ryan et al. Plasma Phys. Control. Fusion 57095008 (2015)

[56] K. Sarkimaki et al. Plasma Phys. Control. Fusion 58125017 (2016)

[57] F.Orain et al. 2013 Physics of Plasmas 20, 102510 (2013)

[58] N.M. Ferraro, Phys.Plasmas 19, 056105 (2012) 